Artículo original

\title{
EVALUACION DE LA CONCENTRACION DE POLVO ATMOSFERICO SEDIMENTABLE Y MATERIAL PARTICULADO (PM2.5, PM10) PARA LA GESTION DE LA CALIDAD DEL AIRE 2017, EN LA CIUDAD DE TACNA
}

EVALUATION OF THE CONCENTRATION OF SEDIMENTABLE ATMOSPHERIC DUST AND PARTICULATED MATERIAL (PM2.5, PM10) FOR AIR QUALITY MANAGEMENT 2017, IN THE CITY OF TACNA

Jeissy Lady Shalom Miranda Casapia ${ }^{1}$ Lizbeth Merma Aruhuanca ${ }^{1}$ Milagros Herrera Rejas ${ }^{2}$

Información del artículo: Recibido: 18/07/2017. Aceptado: 17/12/2017

${ }^{1}$ Ingeniero Ambiental

2.Maestra, docente de la Facultad de Ingeniería, Universidad Privada de Tacna 


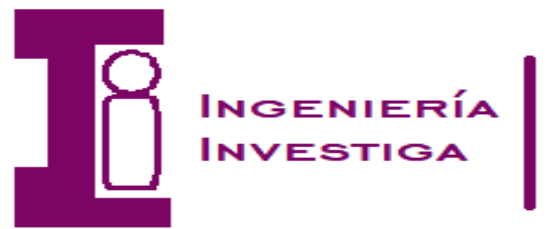

Vol. $1, \mathrm{~N}^{\circ} 1$.

Julio - Diciembre del 2019. 


\section{Resumen}

La ciudad de Tacna no es ajena a la exposición de los agentes contaminantes como las partículas sedimentables que se generan debido a las condiciones atmosféricas estables, al crecimiento urbano, el incremento del tráfico vehicular, la expansión urbana, las calles no pavimentadas, entre otros factores. Mediante el presente trabajo de investigación que se realizó en la ciudad de Tacna, se logró determinar la concentración de polvo atmosférico sedimentable en nuestra ciudad, para lo cual se establecieron 08 estaciones de muestreo donde se aplicó la metodología de Muestreo Pasivo, que consistió en la colocación de placas de vidrio ubicados en el nivel superior de las viviendas y/o instituciones, por un periodo de 04 meses y en ese tiempo se recopiló la información cada 30 días calendarios. Para la ubicación de las estaciones a muestrear se tuvo en cuenta el tipo de vía, densidad poblacional y tráfico; logrando determinar con ello 08 zonas de muestreo localizadas en la zona Centro e Intermedia de la ciudad de Tacna, tomando como referencia el paseo cívico de la ciudad. Los resultados obtenidos del muestreo de partículas sedimentables indican un valor promedio final de 1.07 $\mathrm{mg} / \mathrm{cm} 2 / \mathrm{mes}$ en todas las estaciones de muestreo, el cual se encuentra en el orden de 0.57 $\mathrm{mg} / \mathrm{cm} 2 / \mathrm{mes}$ por encima de los valores guía para partículas atmosféricas sedimentables de la Organización Mundial de la Salud - OMS, fijado en $0.50 \mathrm{mg} / \mathrm{cm} 2 / \mathrm{mes}$. De acuerdo a los resultados obtenidos se logró determinar 3 puntos con mayor grado de significancia, en los cuales se monitoreó el PM2.5 y PM10 con la estación móvil donde se aplicó la metodología de Monitoreo Automático.

Palabras Claves: Calidad de aire, material particulado

\section{Abstract}

The city of Tacna is no stranger to the exposure of pollutants such as sedimentable particles that are generated due to stable weather conditions, urban growth, increased vehicular traffic, urban expansion, unpaved streets, among other factors. . Through the present research work that was carried out in the city of Tacna, it was possible to determine the concentration of sedimentable atmospheric dust in our city, for which 08 sampling stations were established where the Passive Sampling methodology was applied, which consisted of the placement of glass plates located on the upper level of homes and / or institutions, for a period of 04 months and at that time the information was collected every 30 calendar days. For the location of the stations to be sampled, the type of road, population density and traffic were taken into account; managing to determine with it 08 sampling zones located in the Center and Intermediate zone of the city of Tacna, taking as reference the civic walk of the city. The results obtained from the sampling of sedimentable particles indicate a final average value of $1.07 \mathrm{mg} / \mathrm{cm} 2 / \mathrm{month}$ in all sampling stations, which is in the order of $0.57 \mathrm{mg} / \mathrm{cm} 2 /$ month above the guide values for atmospheric particles sediments of the World Health Organization - WHO, set at $0.50 \mathrm{mg} / \mathrm{cm} 2 /$ month. According to the results obtained, it was possible to determine 3 points with a greater degree of significance, in which the PM2.5 and PM10 were monitored with the mobile station where the Automatic Monitoring methodology was applied.

Key Words: Air quality, particulate material

\section{INTRODUCCIÓN}

La ciudad de Tacna presenta un elevado potencial de desarrollo, concentra cerca de 321 mil 351 habitantes, 40 mil vehículos y 17 mil 339 empresas con actividades de comercio, en la base de un área urbana que se extiende sobre una superficie de 16 mil 075 Kilómetros2 (INEI, 2016). Unas expansiones urbanas sin planeación generan el Material Particulado teniendo además un 
acelerado proceso de erosión de los suelos, que han provocado un deterioro ambiental de considerable magnitud. El Material Particulado (MP) es una mezcla compleja de productos químicos y/o elementos biológicos, como metales, sales, materiales carbonosos, compuestos orgánicos volátiles (COV), hidrocarburos aromáticos policíclicos (HAP) y endotoxinas que pueden interactuar entre sí formando otros compuestos (Billet et al., 2007). Está determinado por la forma y densidad de la partícula pudiendo ser clasificadas como finas y gruesas (García, 2002; Bell et al., 2004). Un instrumento estratégico de gestión ambiental que monitoree la calidad del aire, es la evaluación de concentración de Material Particulado (PM2.5 y PM10), que se encuentra establecido en el Estándar de Calidad Ambiental (ECA) para Aire de la legislación peruana y las partículas atmosféricas sedimentables que se encuentra referenciado por la Organización Mundial de la Salud (OMS). Se hace necesario generar políticas y proyectos de mejoramiento de la calidad de aire para proteger la salud de los habitantes. (Cesar Augusto, Universidad de Caldas. 2012)

Según información proporcionada por el Servicio Nacional de Meteorología e Hidrología (SENAMHI), durante el mes de diciembre del año 2012 en Lima Metropolitana, la concentración promedio de Polvo Atmosférico Sedimentable - PAS ( $\Phi<100$ micrómetros) llegó a un promedio de $13,1 \mathrm{t} / \mathrm{km} 2 / \mathrm{mes}$, siendo inferior en 7,1\% a lo registrado en similar mes del año anterior que fue $14,1 \mathrm{t} / \mathrm{km} 2 /$ mes y en 2,6 veces el valor considerado como tolerable por la Organización Mundial de la Salud (OMS).(SENAMHI, 2012). En una valoración de la Campaña de Monitoreo Pasivo De Gases en la Ciudad de Ambato Para el Diseño del Sistema de Calidad del Aire de la Ciudad en Ecuador, encontraron que todos los parámetros estaban bajo los límites establecidos, siendo el dióxido de nitrógeno el que más se acercaba a estos. Se recomendó colocar una estación de monitoreo activo/automático que midan las concentraciones de monóxido de carbono, material particulado y parámetros meteorológicos (Chachapoya, 2015). En el área metropolitana de Costa Rica, durante el 2012, encontraron que los niveles de materia orgánica y carbono elemental (OM + EC) resultaron ser máximos en $\mathrm{HE}-01$ y $\mathrm{BE}-01$, que representan entre $53 \%$ y $56 \%$ de la masa total de partículas PM10. Estos resultados muestran la importancia del aporte de los procesos de combustión a la composición de las partículas finas. Los metales traza representan la contribución más pequeña para todos los sitios de monitoreo (Herrera, Rojas, Beita, Rodríguez, \& Argüello, 2015). Se compararon dos métodos gravimétricos, que permiten cuantificar la concentración de Polvo Atmosférico Sedimentable; los cuales son el Método de Bergerhoff y el Método de las Placas Receptoras, realizándose en las Instalaciones del Campus de la Universidad de El Salvador, Sede Central. Concluyen que las concentraciones están directamente relacionadas a las estaciones de año. (Escobar \& Aquino, 2012). Se monitorearon los niveles de PM2.5 en la zona suroeste (Santa Catarina) y el cruce de las avenidas Madero y Gonzalitos (Centro). Se obtuvo como resultados que durante el periodo de muestreo se detectaron en ambas zonas Benzo(a) Antraceno, Criseno, Benzo(b) Fluoranteno, Benzo(k)Fluoranteno, Benzo(a)Pireno, Benzo(ghi)Perileno, Naftaleno, Acenafteno y Antraceno. Cabe recordar que los 6 PAH's cuantificados son los que están más asociados al desarrollo de cáncer pulmonar (Santiago, 2009). En el Valle de Aburrá Antioquia -Colombia, analizan la calidad del aire en el período 2001-2007. Las concentraciones de partículas en suspensión total (PST) y de partículas respirables (PM10) se encontraron elevadas a niveles que exceden en $200-400 \%$ 
los límites de precaución definidos por la Organización Mundial de la Salud (Bedoya \& Martínez, 2008). También en la Ciudad De Medellín se buscó la relación Entre las Partículas Finas (PM2.5) y Respirables (PM10) observándose una correlación positiva y lineal entre los dos parámetros. (Londoño \& Vasco, 2008).

En la ciudad de Moyobamba, Perú, desde el mes Octubre del 2012 al mes Enero del 2013, se logró determinar el grado de partículas sedimentables en dicha ciudad. Se determinó la existencia de una relación directa entre las condiciones meteorológicas y la generación de partículas sedimentables concluyendo que existe un problema de contaminación ambiental. (Coral, 2012). En la Universidad Nacional de San Marcos de Lima Perú, mediante el monitoreo ambiental se ubicaron 23 puntos de medición, obteniéndose valores por encima de los recomendados en diferentes puntos de la universidad (Huatuco \& Romero, 2011). En la Zona Metropolitana de Lima Callao (Agosto - 2008) se observó que las concentraciones de SO2 y NO2 obtenidas con los tubos pasivos son menores que los obtenidos con los analizadores, las diferencias son $30 \%$ para el SO2 y $86 \%$ para NO2. Las concentraciones de los contaminantes de material particulado PM10 y PM2.5 muestran comportamiento diferente en las horas de ocurrencia de las máximas bimodales. (Jeronimo Garcia V., 2008). En Bogotá, se realizó un análisis de los registros contenidos en la Red de Monitoreo de la Calidad del Aire. Los resultados sugieren que las concentraciones atmosféricas de material particulado en la ciudad tienden a encontrarse muy por encima de los niveles sugeridos por las normas de calidad del aire (Gaitán, Cancino, \& Behrentz, 2007). En la evaluación de las partículas suspendidas totales (PST) y partículas respirables (PM10) en la zona de Guayabal, Medellín, Colombia, las concentraciones presentaron una tendencia a superar la norma anual, de $100 \mu \mathrm{g} / \mathrm{m} 3$. La fracción PM10/PST arrojó un promedio de 0,62 indicando una participación importante de partículas respirables con respecto al total de partículas suspendidas. (Gómez, Henao, Molina, \& Molina, 2003).

Como información alternativa, se sabe que, en Tacna, las enfermedades cardiovasculares, enfermedades hipertensivas y enfermedades isquémicas del corazón son afecciones del sistema circulatorio que cada año toma mayor relevancia en la mortalidad de la población. (Ambiente, 2013). Patologías asociadas también a calidad ambiental.

\section{OBJETIVOS}

- Conocer la concentración de Polvo Atmosférico Sedimentable utilizando el método de Placas Receptoras en puntos de muestreo ubicados estratégicamente en la zona urbana de la ciudad de Tacna.

- Identificar puntos críticos de contaminación de PAS que superan los valores guía establecidos por la OMS, para realizar monitoreo del PM2.5 y PM10 aplicando metodología activa.

- Proponer estaciones de monitoreo de control de contaminación atmosférica en la ciudad de Tacna para determinar una red de monitoreo de calidad de aire de Tacna, según los resultados obtenidos de la aplicación del método de Placas Receptoras y uso estación móvil (PM2.5 y PM10). 


\section{METODOLOGÍA}

Investigación experimental, mediante manipulación de las variables (Rodriguez, 2005). Prospectiva. La muestra estuvo conformada por 08 puntos que presentan similares condiciones como tránsito vehicular, zonas comerciales y urbanas. Se recopiló información de cada punto de muestreo cada 30 días calendarios por espacio de 120 días. Se midió la concentración mediante técnica de pesado - diferencia de peso. El proceso de datos consistió en el trabajo de campo para las mediciones utilizando laboratorio y gabinete en base a la metodología del muestreo pasivo que determina Partículas Atmosféricas Sedimentables (PAS). Previo al recojo de las muestras se pesaron los sustratos que nos sirvieron como recolectores de muestras en una balanza analítica. Al día 30 se procedió a recolectar las placas, cada uno en sus respectivos sobre rotulados y con su peso inicial que fue previamente registrado antes de ser colocado en el recipiente. Se realizaron 04 recolecciones de muestras, cada 30 días, de los 08 puntos muestreo en el mismo día; siendo el tiempo total de muestreo de 120 días. Las muestras obtenidas en los puntos de muestreo fueron herméticamente cerradas y fueron abiertos en laboratorio para ser pesados en una balanza analítica y por diferencia de peso se conoció el peso de las partículas. Se determinó el peso de las partículas y se proyectó la concentración mediante:

\section{PAS $=$ PS $=\left(\left(\mathrm{mg} / \mathrm{cm}^{2}\right)\right.$ Mes $)=($ Peso Final - Peso Inicial $/$ Área $) 1$ Mes $)$}

El monitoreo automático de partículas en suspensión en el ambiente se realizó a través de métodos gravimétrico. Los equipos utilizados son muestreadores de alto volumen y bajo volumen por tomar medidas de las concentraciones de material particulado menor a 2.5 micrómetros y 10 micrómetros conocidas como PM2.5 y PM10. El equipo de monitoreo de aire automático tipo fue el THERMO SCIENTIFIC - TEOM 1405 del Gobierno Regional de Tacna.

\section{RESULTADOS}

En la Tabla 01 y Fig. 01 se muestra los resultados del muestreo de Partículas Atmosféricas Sedimentables para las estaciones de muestreo E5, E6, E7 y E8, de la Zona 1 en el centro de la ciudad de Tacna, así como los valores promedio en esta zona (Fig. 02).

\begin{tabular}{|c|c|c|c|c|c|}
\hline \multirow{2}{*}{$\begin{array}{l}\text { ZONA } 1 \\
\text { MES/FECHA }\end{array}$} & \multicolumn{5}{|c|}{ PARTE CENTRICA DE LA CIUDAD DE TACNA } \\
\hline & E5 & E6 & E7 & E8 & PROMEDIO \\
\hline FEBRERO & 0.45 & 0.16 & 1.76 & 2.44 & 1.21 \\
\hline MARZO & 0.44 & 0.49 & 1.10 & 1.33 & 0.84 \\
\hline ABRIL & 0.33 & 0.67 & 0.78 & 1.48 & 0.82 \\
\hline MAYO & 0.40 & 0.85 & 0.98 & 2.01 & 1.06 \\
\hline SUMATORIA & 1.63 & 2.17 & 4.62 & 7.27 & \\
\hline PROMEDIO & 0.41 & 0.54 & 1.16 & 1.82 & 0.98 \\
\hline
\end{tabular}




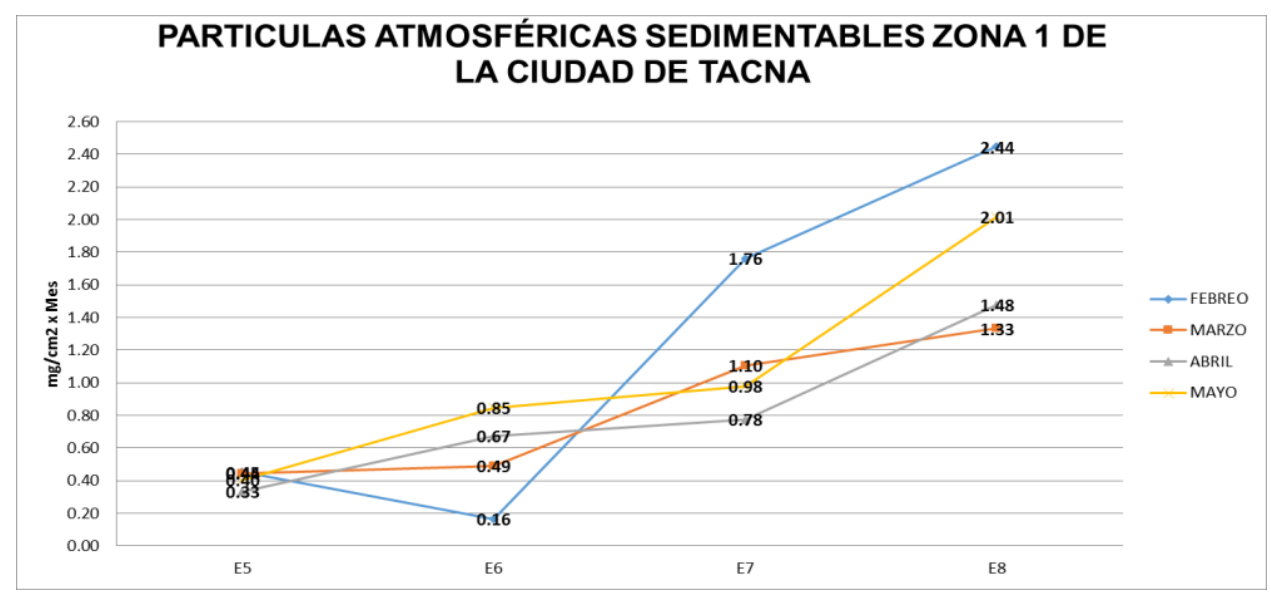

Fig. 01: Resultados del muestreo de Partículas Atmosféricas Sedimentables en la Zona 1: Centro de la Ciudad de Tacna en $\mathrm{mg} / \mathrm{cm} 2 / \mathrm{mes}$

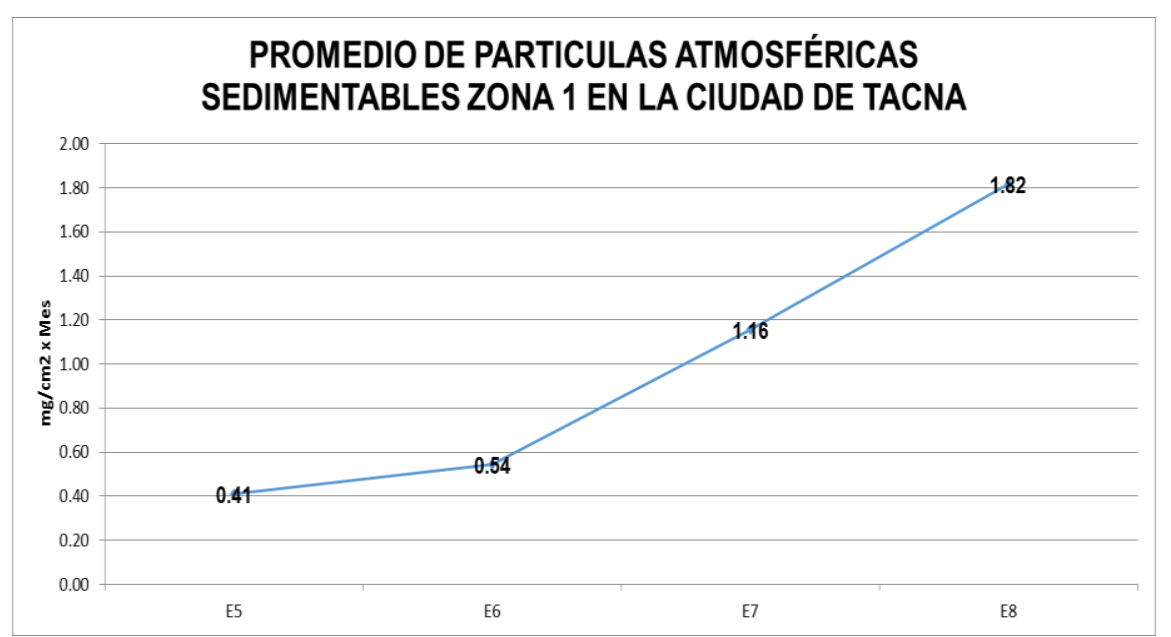

Fig. 02: Resultados promedio del muestreo de Partículas Atmosféricas Sedimentables en la Zona 1: Céntrica de la Ciudad de Tacna en $\mathrm{mg} / \mathrm{cm} 2 / \mathrm{mes}$.

La Tabla y Fig. 2 muestra los resultados del muestreo de Partículas Atmosféricas Sedimentables para las estaciones de muestreo E1, E2, E3 y E4, de la Zona 2 - zona intermedia de la ciudad de Tacna.

Tabla 02: Resultados de muestreo de Partículas Atmosféricas Sedimentables en la Zona 2 - zona intermedia de la ciudad de Tacna en $\mathrm{mg} / \mathrm{cm} 2 / \mathrm{mes}$.

\begin{tabular}{lccccc}
\hline ZONA 2 & \multicolumn{5}{c}{ PARTE INTERMEDIA DE LA CIUDAD DE TACNA } \\
MES/FECHA & E1 & E2 & E3 & E4 & PROMEDIO \\
\hline FEBRERO & 1.42 & 0.82 & 3.28 & 2.21 & 1.93 \\
MARZO & 0.24 & 0.06 & 1.01 & 0.65 & 0.49 \\
ABRIL & 0.46 & 0.89 & 1.19 & 0.96 & 0.88 \\
MAYO & 0.18 & 0.97 & 1.32 & 2.74 & 1.30 \\
SUMATORIA & 2.30 & 2.73 & 6.80 & 6.57 & \\
PROMEDIO & 0.57 & 0.68 & 1.70 & 1.64 & 1.15 \\
\hline
\end{tabular}




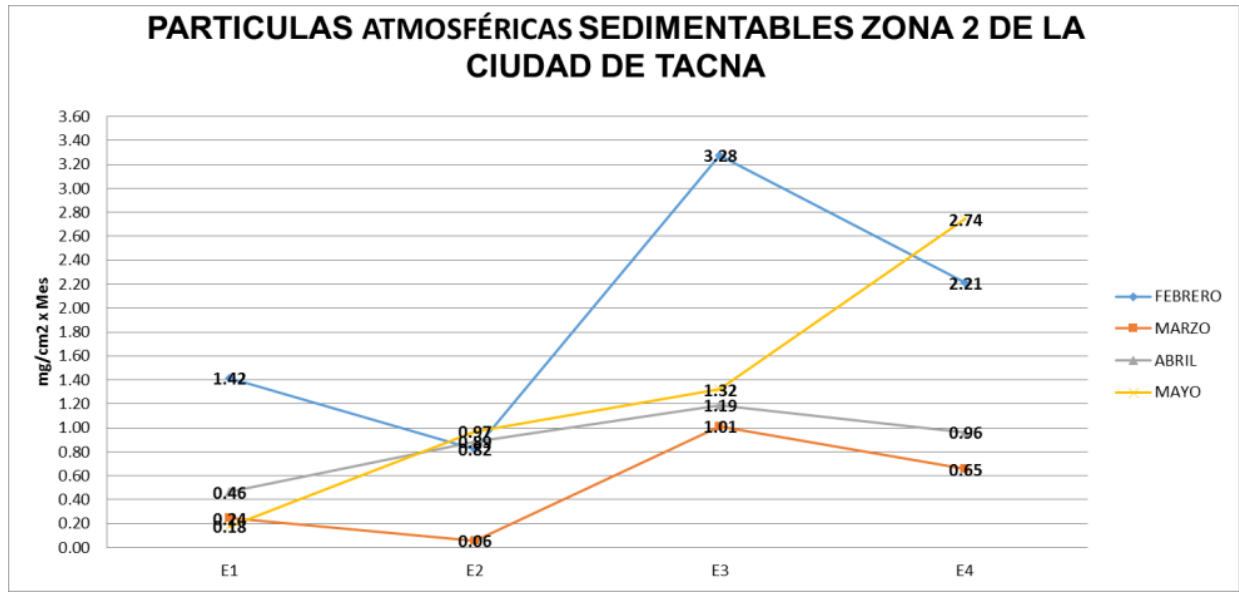

Fig. 03: Resultados del muestreo de Partículas Atmosféricas Sedimentables en la Zona 2: Intermedia de la Ciudad de Tacna en $\mathrm{mg} / \mathrm{cm} 2 / \mathrm{mes}$.

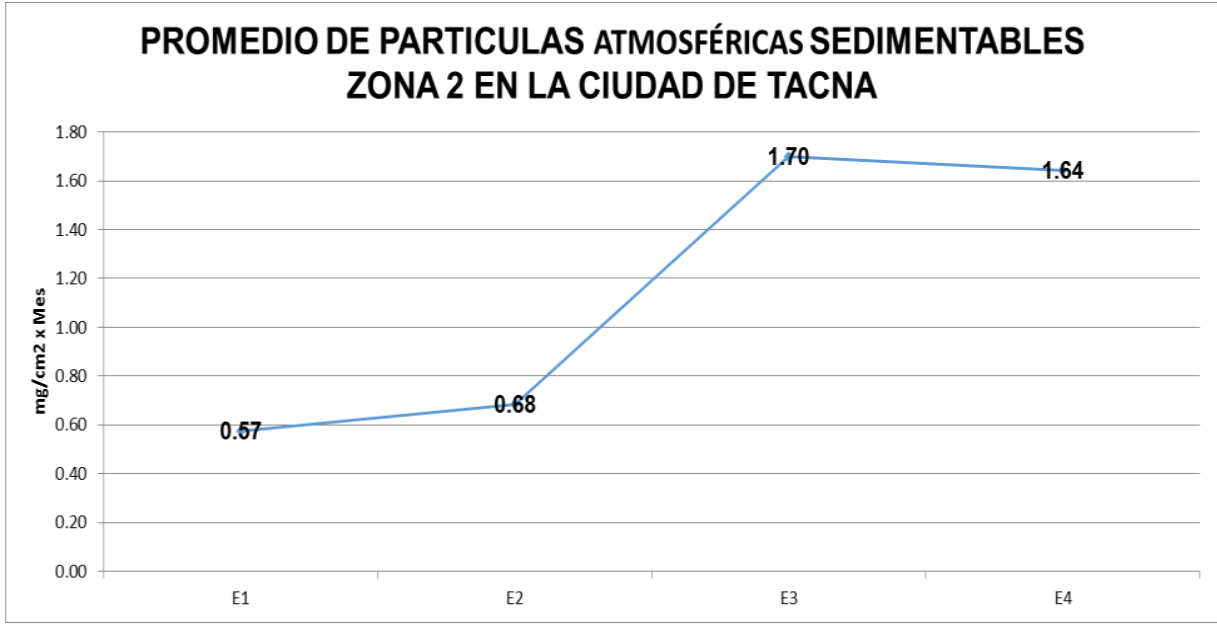

Fig. 04: Resultados promedio del muestreo de Partículas Atmosféricas Sedimentables Zona 2: Intermedia de la Ciudad de Tacna en $\mathrm{mg} / \mathrm{cm} 2 / \mathrm{mes}$.

Las Tabla 03 muestra el análisis de varianza de las zonas establecidas: Zona Centro e Intermedia de la ciudad de Tacna en $\mathrm{mg} / \mathrm{cm} 2 /$ mes.

Tabla 03: Análisis de Varianza de Zonas Establecidas.

\begin{tabular}{ccc}
\hline REPETICIONES & R1= Zona 1 & R2= Zona 2 \\
\hline MES 1 & 1.21 & 1.93 \\
MES 2 & 0.84 & 0.49 \\
MES 3 & 0.82 & 0.88 \\
MES 4 & 1.06 & 1.30 \\
Xi & 3.92 & 4.60 \\
PROMEDIO & 0.98 & 1.15 \\
\hline
\end{tabular}


Los resultados del estudio del material particulado en suspensión (PM2.5 y PM10) en el medio atmosférico fueron obtenidos a través del peso del material particulado con tamaño igual o menor que 2.5 y $10 \mu \mathrm{m}$, respectivamente. Los resultados de los análisis y cálculos para las partículas en suspensión PM2.5 y PM10 se muestran según las estaciones seleccionadas

Estación 3 (E3) - Centro de Salud Leoncio Prado En la Fig. 5 se observa de manera referencial que las concentraciones horarias de partículas PM2.5, en la estación de monitoreo del Centro de Salud Leoncio Prado (E3), durante el periodo de 24 horas, exceden los valores guía de la OMS, fijado en $25 \mu \mathrm{g} / \mathrm{m} 3$, en el horario diurno y nocturno, mas no el ECA de Aire nacional fijado en 50 $\mu \mathrm{g} / \mathrm{m3}$. Finalmente, tenemos que el promedio registrado de $\mathrm{PM} 2.5$, durante el periodo de 24 horas, es de $22 \mu \mathrm{g} / \mathrm{m} 3$, el cual no excede el Estándar de Calidad Ambiental (ECA) del Aire nacional para PM2.5 fijado en $50 \mu \mathrm{g} / \mathrm{m} 3$ ni el valor guía de la OMS, fijado en $25 \mu \mathrm{g} / \mathrm{m} 3$.

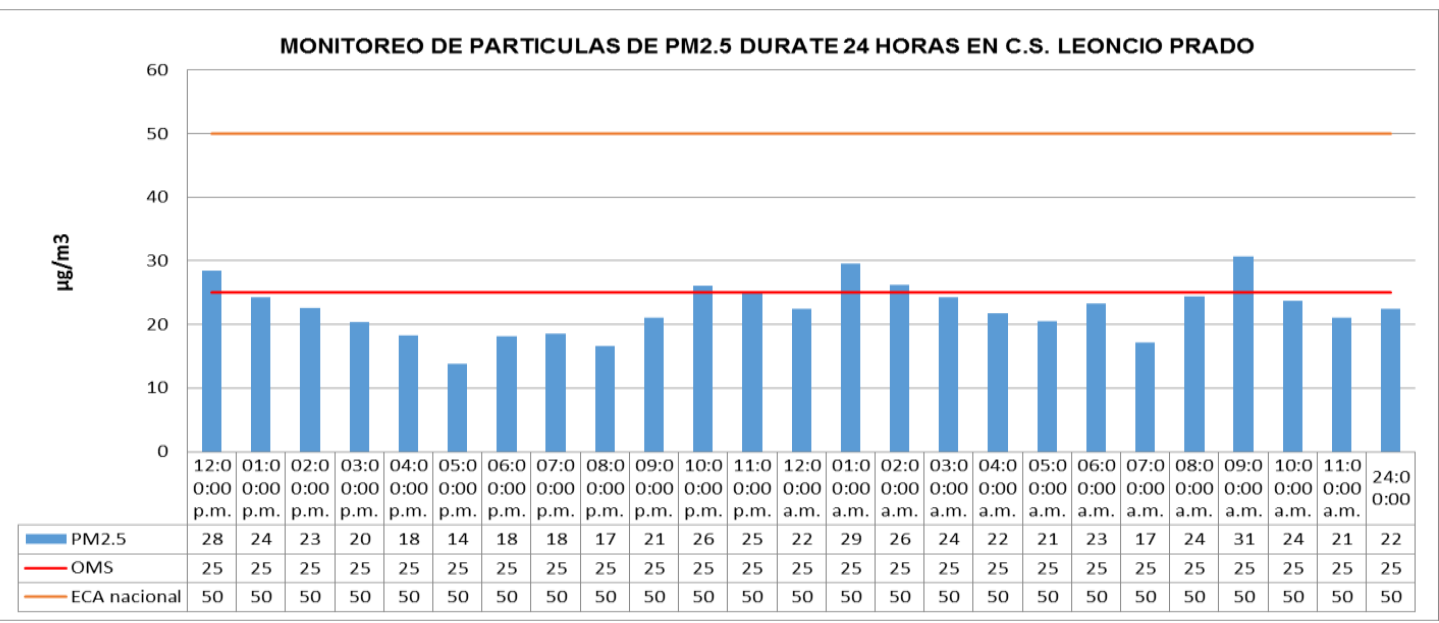

Fig. 5: Monitoreo de partículas PM2.5 durante 24 horas en el Centro de Salud de Leoncio Prado (E3) comparado con los Estándares de Calidad Ambiental (ECA) del Aire nacional y el de la OMS.

En la Fig. 06 se observa de manera referencial que las concentraciones horarias de partículas PM10, en la estación de monitoreo del Centro de Salud Leoncio Prado (E3), durante el periodo de 24 horas, exceden los valores guía de la OMS, fijado en $50 \mu \mathrm{g} / \mathrm{m3}$, en los horarios de las 12:00 p.m., 1:00 p.m., 2:00 p.m., 9:00 p.m. y 10:00 p.m. del día 18 de julio de 2017 y la 1:00 a.m. y 9:00 a.m. del día 19 de julio de 2017, mas no el ECA de Aire nacional fijado en $100 \mu \mathrm{g} / \mathrm{m} 3$. Finalmente, tenemos que el promedio registrado de PM10, durante el periodo de 24 horas, es de $44 \mu \mathrm{g} / \mathrm{m} 3$, el cual no excede el Estándar de Calidad Ambiental (ECA) del Aire nacional para PM10 fijado en $100 \mu \mathrm{g} / \mathrm{m} 3 \mathrm{ni}$ el valor guía de la OMS, fijado en $50 \mu \mathrm{g} / \mathrm{m3}$. 


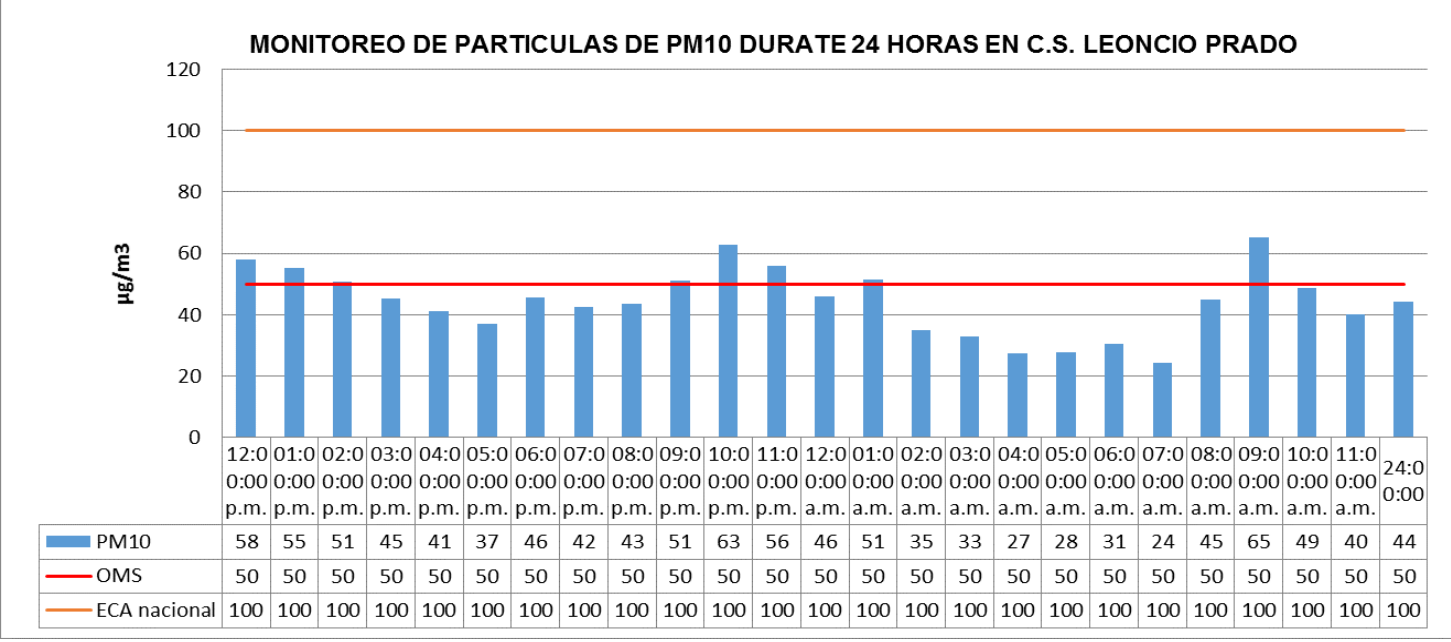

Fig 06: Monitoreo de partículas PM10 durante 24 horas en el Centro de Salud de Leoncio Prado (E3) comparado con los Estándares de Calidad Ambiental (ECA) del aire nacional y los valores guía de la OMS

\section{Estación 7 (E7) - Centro de Post Grado UNJBG}

En la Fig. 07 se observa que de manera referencial las concentraciones horarias de partículas PM2.5, en la estación de monitoreo del Centro de Post Grado de la UNJBG (E7), durante el periodo de 24 horas, excede los valores guía de la OMS, fijado en $25 \mu \mathrm{g} / \mathrm{m3}$, en el horario diurno y nocturno; mientras que en los horarios de las 12:00 p.m. del día 22 de julio de 2017 y las 10:00 a.m. del día 23 de julio de 2017 se excede el ECA de Aire nacional, fijado en $50 \mu \mathrm{g} / \mathrm{m3}$. Finalmente, tenemos que el promedio registrado de PM2.5, durante el periodo de 24 horas, es de $34 \mu \mathrm{g} / \mathrm{m} 3$, el cual no excede el Estándar de Calidad Ambiental (ECA) del Aire nacional para PM2.5 fijado en $50 \mu \mathrm{g} / \mathrm{m} 3$ pero si excede el valor guía de la OMS, fijado en $25 \mu \mathrm{g} / \mathrm{m} 3$.

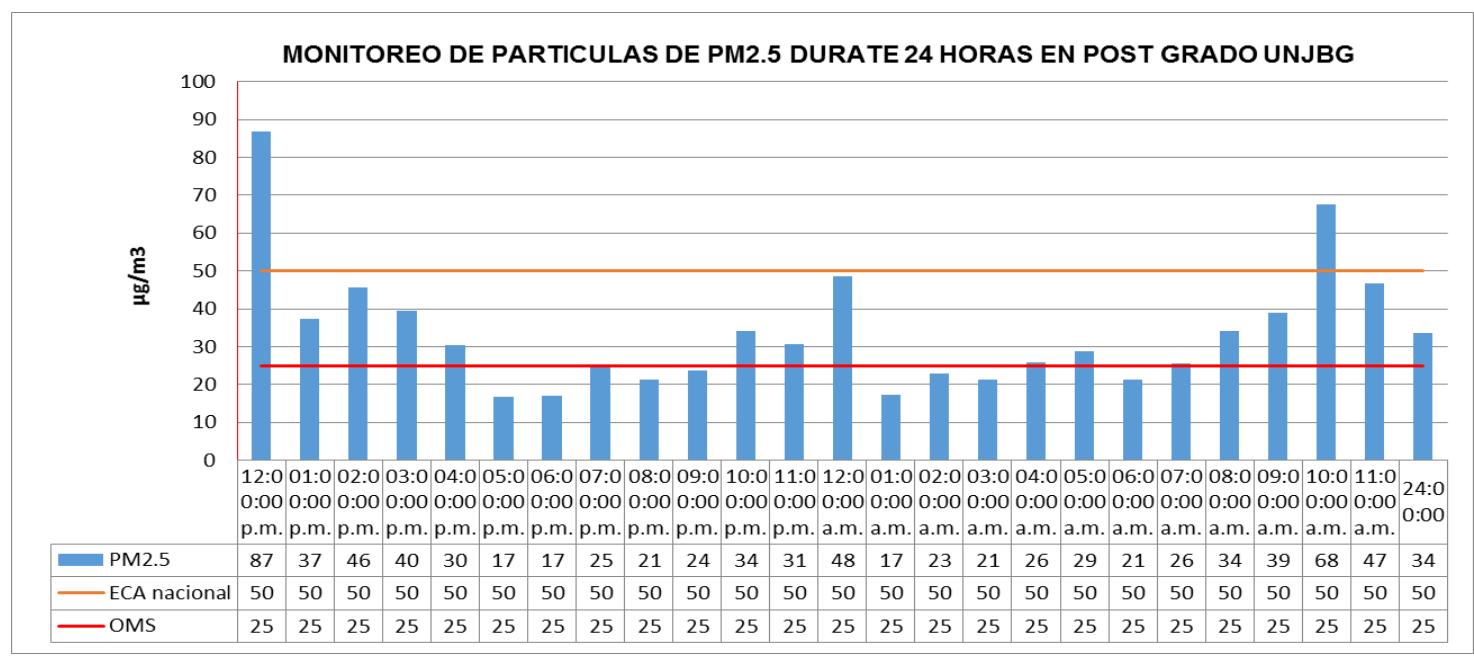

Fig. 07: Monitoreo de partículas PM2.5 durante 24 horas en el Centro de Post Grado de la UNJBG (E7) comparado con los Estándares de Calidad Ambiental (ECA) del Aire nacional y los valores guía de la OMS. 
En la Fig. 08 se observa de manera referencial que las concentraciones horarias de partículas PM10, en la estación de monitoreo del Centro de Post Grado de la UNJBG (E7), durante el periodo de 24 horas, exceden los valores guía de la OMS, fijado en $50 \mu \mathrm{g} / \mathrm{m3}$, en los horarios de las 12:00 p.m. a 4:00 p.m., 7:00 p.m., 10:00 p.m. y 11:00 p.m. del día 22 de julio de 2017, y en los horarios de las 12.00 a.m. y de 8:00 a.m. a 11:00 a.m. del día 23 de julio de 2017; mientras que en los horarios de las 12:00 p.m. del día 22 de julio de 2017 y las 10:00 a.m. del día 23 de julio de 2017 se excede el ECA de Aire nacional, fijado en $100 \mu \mathrm{g} / \mathrm{m} 3$. Finalmente, tenemos que el promedio registrado de PM10, durante el periodo de 24 horas, es de $62 \mu \mathrm{g} / \mathrm{m} 3$, el cual no excede el Estándar de Calidad Ambiental (ECA) del Aire nacional para PM10 fijado en $100 \mu \mathrm{g} / \mathrm{m} 3$ pero si excede el valor guía de la OMS, fijado en $50 \mu \mathrm{g} / \mathrm{m} 3$.

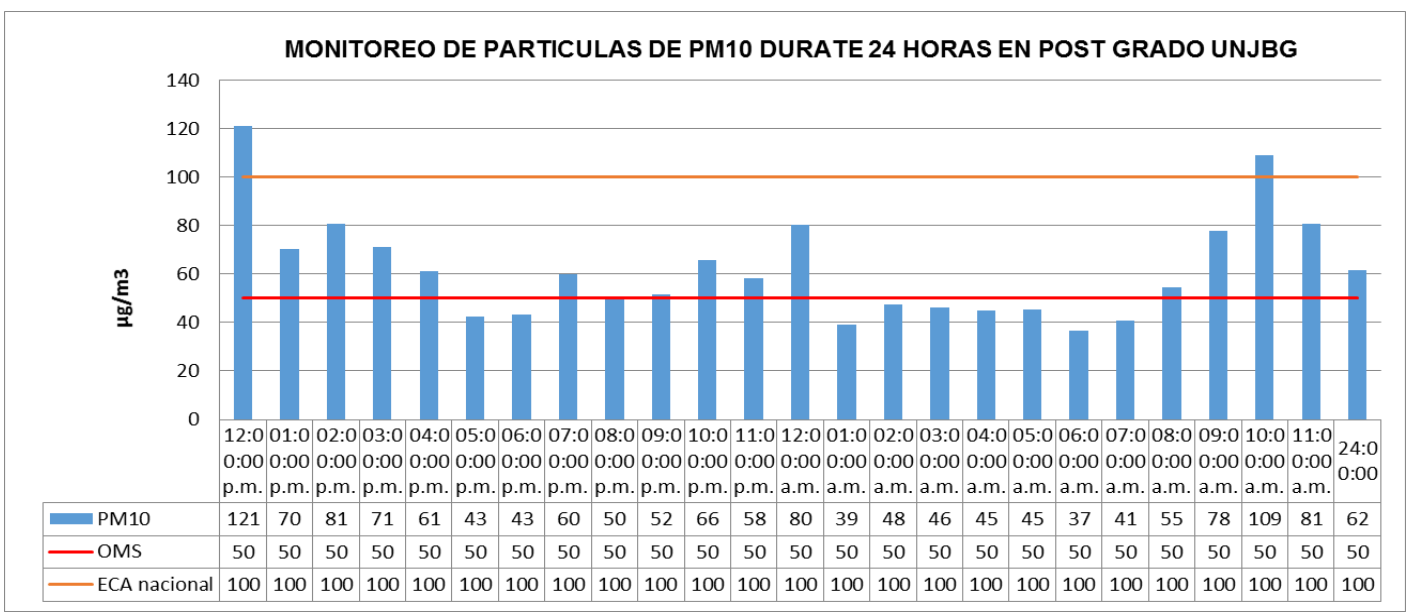

Fig. 08: Monitoreo de partículas PM10 durante 24 horas en el Centro de Post Grado de la UNJBG (E7) comparado con los Estándares de Calidad Ambiental (ECA) del aire nacional y los valores guía de la OMS.

Estación 8 (E6) - Gran Hotel (SERPOST)

En la Fig. 09 se observa de manera referencial que las concentraciones horarias de partículas PM2.5, en la estación de monitoreo del Gran Hotel Tacna (Serpost) (E8), durante el periodo de 24 horas, exceden los valores guía de la OMS, fijado en $25 \mu \mathrm{g} / \mathrm{m3}$, entre las 12:00 p.m. a 4:00 p.m. y las 6:00 p.m. del día 14 de julio de 2017, y entre las 8:00 a.m. a 11:00 a.m. del día 15 de julio de 2017; mientras que a las 12:00 p.m. y 3:00 p.m. del día 22 de julio de 2017 exceden el ECA de Aire nacional fijado en $50 \mu \mathrm{g} / \mathrm{m} 3$. Finalmente, tenemos que el promedio registrado de PM2.5, durante el periodo de 24 horas, es de $26 \mu \mathrm{g} / \mathrm{m} 3$, el cual no excede el Estándar de Calidad Ambiental (ECA) del Aire nacional para PM2.5 fijado en $50 \mu \mathrm{g} / \mathrm{m} 3$ pero si excede el valor guía de la OMS, fijado en $25 \mu \mathrm{g} / \mathrm{m} 3$. 


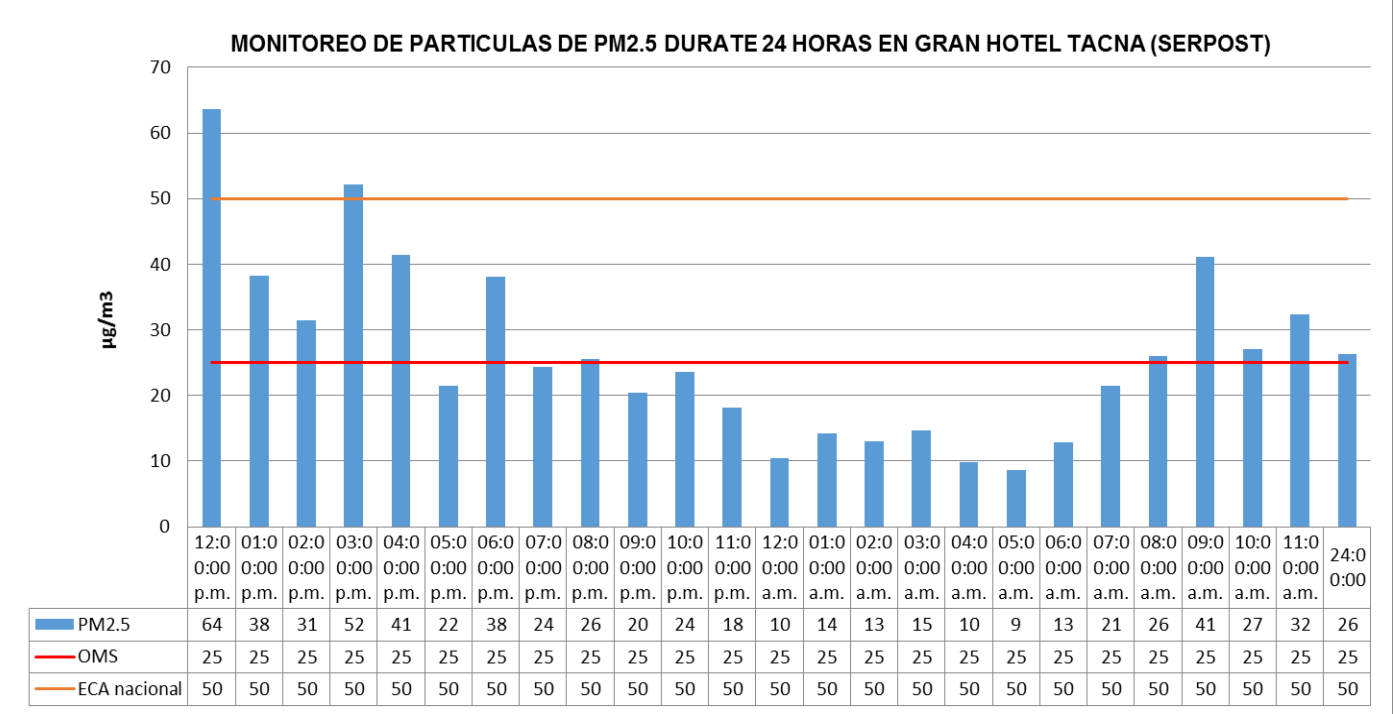

Fig. 09: Monitoreo de partículas PM2.5 durante 24 horas en el Gran Hotel Tacna (SERPOST) (E8) comparado con los Estándares de Calidad Ambiental (ECA) del Aire nacional y los valores guía de la OMS.

En la Fig. 10 se observa referencialmente que las concentraciones horarias de partículas PM10, en la estación de monitoreo del Gran Hotel Tacna (SERPOST) (E8), durante el periodo de 24 horas, exceden los valores guía de la OMS, fijado en $50 \mu \mathrm{g} / \mathrm{m3}$, en el horario de las 12:00 p.m., 3:00 p.m., 4:00 p.m. y 6:00 p.m. del día 14 de julio de 2017 y las 9:00 a.m. del día 15 de julio de 2017, mas no el ECA de Aire nacional fijado en $100 \mu \mathrm{g} / \mathrm{m} 3$. Finalmente, tenemos que el promedio registrado de PM10, durante el periodo de 24 horas, es de $38 \mu \mathrm{g} / \mathrm{m3}$, el cual no excede el Estándar de Calidad Ambiental (ECA) del Aire nacional para PM10 fijado en $100 \mu \mathrm{g} / \mathrm{m} 3$ y el valor guía de la OMS fijado en $50 \mu \mathrm{g} / \mathrm{m} 3$.

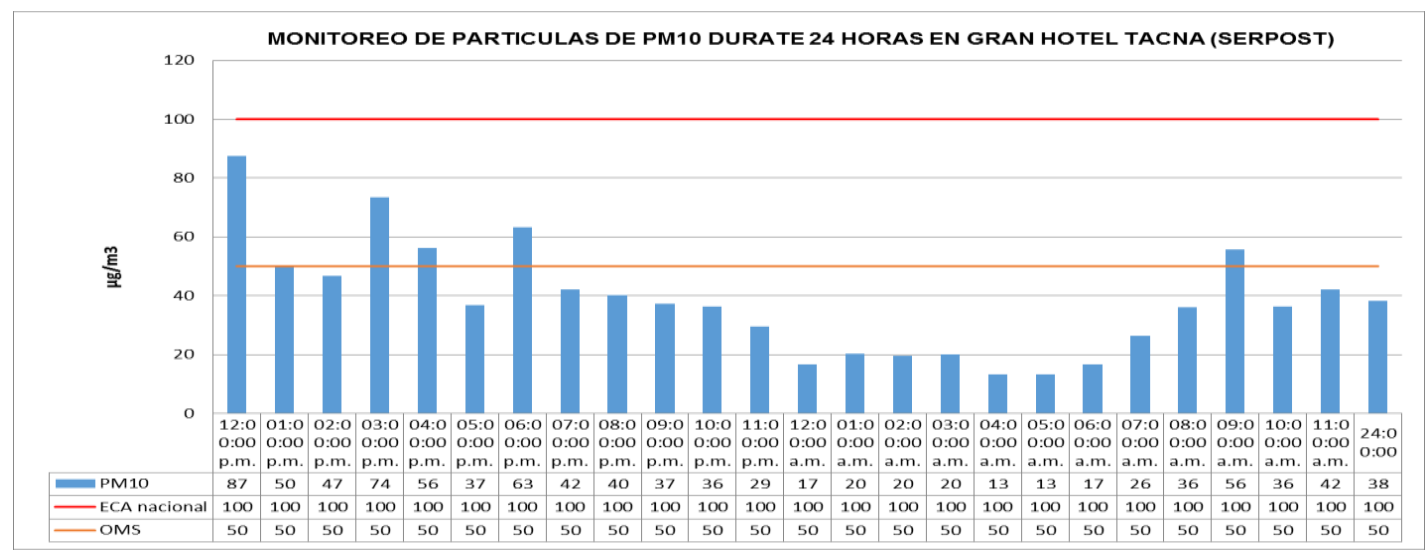

Fig. 10: Monitoreo de partículas PM10 durante 24 horas en Gran Hotel Tacna (SERPOST) (E8) comparado con los Estándares de Calidad Ambiental (ECA) del aire nacional y los valores guía de la OMS Monitoreo del PM10 de 24 horas en Gran Hotel Tacna.

En el Fig. 11se muestra la Rosa de Vientos de la estación meteorológica Jorge Basadre, ubicada 
en la localidad de Tacna, para el periodo comprendido desde el mes de febrero hasta el mes de mayo de 2017, indicándonos que la componente del viento Suroeste (SW) es la que mayormente predomina, con intensidades de viento de hasta $8.8 \mathrm{~m} / \mathrm{s}$ en esa misma dirección, estando esta componente del $\mathrm{SW}$ asociada con el viento sinóptico del Anticiclón del Pacífico Sur que ingresa por el litoral costero de Tacna, induciendo una brisa mar - tierra en el sentido SW - NE, el cual transporta el material particulado en el ambiente en el sentido de la brisa mar - tierra hacia la ciudad de Tacna.

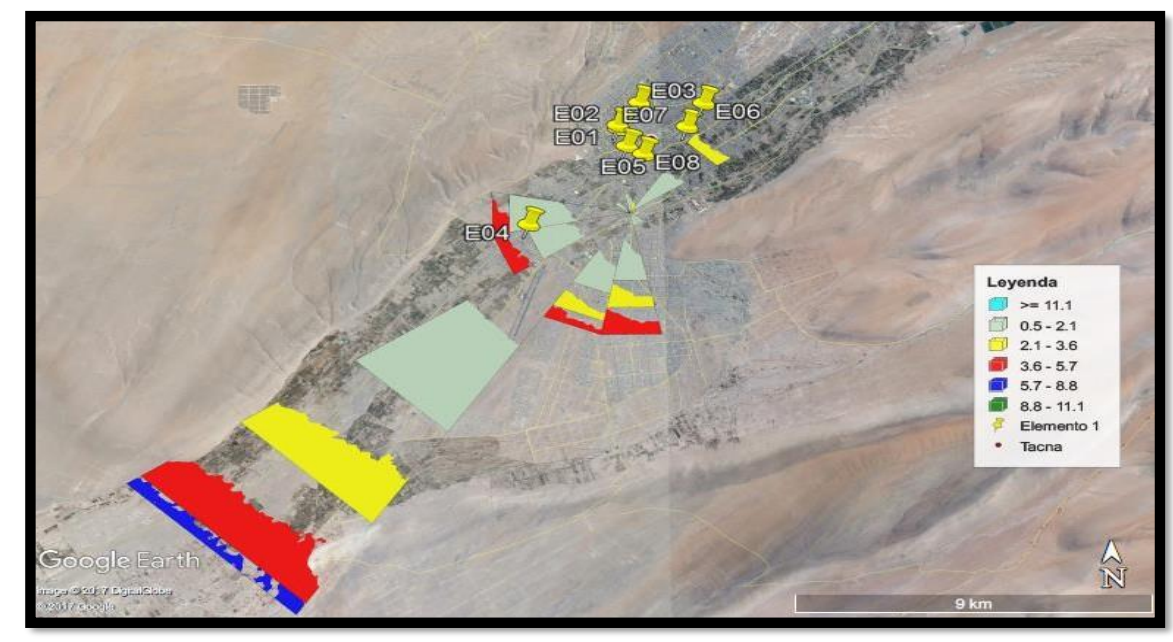

Fig. 11: Mapa de Rosa de Viento y Estaciones de muestreo para el PAS y Monitoreo de PM2.5 y PM10 por el método pasivo en la ciudad de Tacna.

\section{DISCUSIÓN}

Como referencia, la Organización Mundial de la Salud - OMS ha establecido valores guía para la concentración de Polvo Atmosférico Sedimentable (PAS) en el aire de $0.5 \mathrm{mg} / \mathrm{cm} 2 / \mathrm{mes}$. La distribución de la concentración de PAS en la ciudad de Tacna sobrepasa estos valores establecidos, tal es el caso de La Zona 2 = Zona Intermedia de la Ciudad de Tacna, que supera el valor guía de la OMS con un valor de $0.65 \mathrm{mg} / \mathrm{cm} 2 / \mathrm{mes}$; mientras que en la Zona 1 = Centro de la ciudad de Tacna, se encuentra por debajo del valor guía de la OMS con un valor de 0.48 $\mathrm{mg} / \mathrm{cm} 2 / \mathrm{mes}$. Este comportamiento diferenciado se debe a las condiciones que presenta la Zona 2 = Zona Intermedia es decir la existencia de alta densidad habitacional y tránsito intermedio; mientras que las condiciones de la Zona 01: Centro de la ciudad de Tacna, presenta la mayor parte de las calles pavimentadas, disminuyendo con ello la remoción de partículas.

En las dos zonas de Monitoreo (Zona 1 y Zona 2) se observa que en el mes de Febrero de 2017 se presenta la mayor concentración de PAS en el aire, este comportamiento guarda relación con la estación de verano, donde se registran altas temperaturas y velocidad del viento ligeramente intensos, según los datos meteorológicos obtenidos de la estación de Tacna, en comparación con los registros meteorológicos del resto de meses monitoreados; lo que demuestra que las condiciones meteorológicas juegan un papel importante en el control natural de las PAS.

Como principales fuentes de generación de PAS en el aire, tenemos al Transporte Urbano, calles sin Pavimentar, Acumulación y Quema de Residuos Sólidos y biomasa de los campos de cultivo, 
Desarrollo de Actividades económicas, principalmente las dedicadas a la comercialización de productos, lo que nos lleva a determinar la urgente necesidad de mejorar el parque automotor, mejorar las infraestructura de las emisiones que genera los restaurantes que utilizan carbón y zonificación urgente de la ciudad.

Según la Organización Mundial de la Salud, al sobrepasar los valores guía establecidos para la concentración de PAS de $0.5 \mathrm{mg} / \mathrm{cm} 2 / \mathrm{mes}$, como es el caso de los resultados obtenidos en ambas zonas de la ciudad de Tacna (Zona 1 y Zona 2) con un valor de $1.07 \mathrm{mg} / \mathrm{cm} 2 / \mathrm{mes}$ como Promedio Total Final, podemos inferir que la población está expuesto a presentar afecciones respiratorias, oftalmológicas, dérmicas, influenciando en su desempeño diario, economía y estabilidad emocional.

El monitoreo de partículas PM2.5 y PM10 en la ciudad de Tacna se realizó en una etapa, es decir sólo en una época del año, en invierno, siendo el mes elegido el mes de julio. Para el desarrollo del presente monitoreo de partículas para una sola época se usó un equipo automático de propiedad del Gobierno Regional de Tacna (GORE). La estrategia de monitoreo utilizada por las normas legales peruanas indican el monitoreo de 24 horas continuas cada 6 meses, y los datos que de allí resulten son "suficientes" en términos legales. Sin embargo, la caracterización del aire, en el parámetro que sea, para obtener validez científica, académica y estadística requiere programas de monitoreo más extensos. En el presente estudio se han monitoreado 3 días, con frecuencia mensual para calcular el valor de las concentraciones de material particulado menor a 2.5 micrómetros y 10 micrómetros, PM2.5 y PM10, por lo que puede considerarse los resultados obtenidos más claros o próximos. Se debe tener en cuenta que las Partículas Atmosféricas Sedimentables, sedimentan más rápido que el material particulado, ya que el material particulado por ser más fino demora en sedimentar, por tal motivo la diferencia de resultados al momento de aplicar ambas metodologías. Si bien es cierto que el monitoreo activo se realiza por 24 horas, establecido por la norma nacional, se evidencia una variación de la concentración en distintas horas.

\section{CONCLUSIONES}

El resultado promedio final es de $1.07 \mathrm{mg} / \mathrm{cm} 2 / \mathrm{mes}$ de PAS, el cual sobrepasa en 0.57 $\mathrm{mg} / \mathrm{cm} 2 / \mathrm{mes}$. Existe relación directa entre las condiciones meteorológicas y la generación de partículas sedimentables, concentración de PAS con un valor de $1.93 \mathrm{mg} / \mathrm{cm} 2 / \mathrm{mes}$. En las estaciones de monitoreo E3, E7 y E8 se encuentran por debajo del ECA para Aire de, respectivamente de acuerdo al D.S. N ${ }^{\circ} 003-2017-M I N A M$. De acuerdo a los resultados promedio obtenidos del monitoreo de PM2.5 y PM10, concluimos que superan los valores guía 25 y 50 $\mu \mathrm{g} / \mathrm{m} 3$ diario de PM2.5 y PM10 brindado por OMS.

\section{RECOMENDACIONES}

- Sugerir al Gobierno Regional y/o la Municipalidad Provincial de Tacna considerar la importancia de incrementar los entornos ecológicos como las áreas verdes con la finalidad de mitigar el Polvo Atmosférico Sedimentable.

- La Municipalidad Provincial de Tacna, debe establecer un sistema de vigilancia de la calidad del aire que sea liderado por la Comisión del Medio Ambiente, a través de sus organizaciones de base más representativas, asesorada por la coordinación inter institucional, este sistema podría constar de una estación de monitoreo fija.

- Considerar como puntos fijos de monitoreo continuo de PM2.5 y PM10 en las estaciones 
E3, E7 y E8 por ser las que presentaron mayor concentración de partículas atmosféricas sedimentables, previo muestreo pasivo y ser considerados puntos críticos.

\section{REFERENCIAS BIBLIOGRÁFICAS}

Ambiente, D. G. (2013). Plan de Acción para la mejora de la calidad del aire en la zona de atención Prioritoria de la Cuenca Atmosférica de Tacna. Tacna.

Bances Estela, V. M. (2003). Contaminación Atmosférica y su Impacto Ambiental en la Ciudad de Moyobamba. San Martín.

Bedoya, J., \& Martínez, E. (12 de Diciembre de 2008). Calidad del Aire en el Valle de Aburrá Antioquia - Colombia. Obtenido de Scielo: http://www.scielo.org.co/pdf/dyna/v76n158/a01v76n158.pdf

Benza, J. C. (1982). Métodos estadísticos para la investigación. En J. C. Benza,

Métodos estadísticos para la investigación (pág. 643). Lima: Milagros.

Benza, J. C. (1982). Metodos estaditicos para la investigacion. En J. C. Benza,

Metodos estaditicos para la investigacion. Lima: Milagros.

BioEnciclopedia. (28 de Marzo de 2014). Contaminación atmosférica. Recuperado el 20 de Julio de 2014, de Contaminación atmosférica:

http://www.bioenciclopedia.com/contaminacion-atmosferica/

Chachapoya, M. P. (2015). “Valoración de la Campaña de Monitoreo Pasivo De Gases en la Ciudad de Ambato Para el Diseño del Sistema de Calidad del Aire de la Ciudad 2013 2014". Quito.

Coral, F. R. (2012). "Determinación del Grado de Partículas Atmosféricas Sedimentables, Mediante el Método de Muestreo Pasivo, Zona Urbana - Ciudad de Moyobamba, 2012". Moyobamba.

Cotrina, J. S. (2008). Evaluación de la Contaminación Atmosférica en la zona metropolitana de Lima-Callao. Lima.

Escobar, A. M., \& Aquino, D. M. (2012). “Comparación de los Métodos De Bergerhoff y Placas Receptoras Para la Cuantificación de Polvo Atmosférico Sedimentable". San Salvador.

Gaitán, M., Cancino, J., \& Behrentz, E. (1 de Octubre de 2007). Análisis del estado de la calidad del aire. Obtenido de Scielo: http://www.scielo.org.co/pdf/ring/n26/n26a11.pdf

Gómez, A., Henao, E., Molina, E., \& Molina, F. (2003). "Evaluacion de las particulas suspendidas totales (PST) y particulas respirables (PM10) en la Zona Guayaquil,Medellin,Colombia". Facultad de Ingenieria No. 30 ., 24-33.

Herrera, J., Rojas, J. F., Beita, V. H., Rodríguez, A., \& Argüello, D. S. (2015). “Concentración y composición química de partículas PM10 en el área metropolitana de Costa Rica en 2012". Revista de Caiencias Abientales Tropical Journal of Environmental, 39-53.

Huatuco, R. M., \& Romero, A. V. (2011). "Contaminacion del Aire por Material Particulado en la ciudad Universitaria - UNMSM". En Proyecto Libro Digital (págs. 1-14). Lima: Guzlop.

INEI. (26 de Agosto de 2016). Departamento de Tacna cuenta con una población de 346 mil habitantes. Obtenido de Departamento de Tacna cuenta con una población de 346 mil habitantes: https://www.inei.gob.pe/prensa/noticias/departamento-de-tacna-cuentacon- una-poblacion-de-346-mil-habitantes-9270/

Jeronimo Garcia V., J. T. (2008). "Evaluación del grado de contaminación del aire en el Centro Histórico de Lima". Anales Científicos de la Universidad Nacional Agraria La Molina, 1-11.

Korc Marcelo, F. F. (2000). El Proceso de Fijación y Revisión de Normas de calidad del aire. En F. F. Korc Marcelo, El Proceso de Fijación y Revisión de Normas de calidad del aire. Lima: CEPIS-OPS.

Londoño, C. A., \& Vasco, G. J. (2008). “Relación Entre las Partículas Finas (PM2.5) y Respirables 
(PM10) en la Ciudad De Medellín". Revista Ingenierías Universidad de Medellín, 23-42. Molina, M. (2001). Estrategia Integral de Gestión de la Calidad del aire. En M. Molina, Estrategia Integral de Gestión de la Calidad del aire. México.

Ocmin, Y. (2013). Monitor de partículas TEOM 1405DF. Lima, Lima, Perú: ENVIROEQUIP SAC. OEFA. (s.f.). Equipos de Medición de la calidad de aire. Lima, lima, Perù: Minam.

Rodriguez, E. (2005). Metodología de la Investigación.

Rodríguez, V. (17 de Mayo de 2017). Diferencia entre adsorción y absorción. Recuperado el 24 de Julio de 2017, de Diferencia entre adsorción y absorción: https://diferencias.eu/entre-adsorcion-y-absorcion/

Sandoval, H. (2000). Contaminación y contaminantes, aspectos científicos, teoóricos y prácticos. En H. S. L, Contaminación y contaminantes, aspectos científicos, teoóricos y prácticos. Lima.

Santiago, O. G. (2009). Determinación del contenido de PAH's en partículas PM2.5 en una zona de alto tráfico vehicular y otra con potencial exposición industrial del Área Metropolitana de Monterrey. Monterrey.

SENAMHI. (2012). Servicio Nacional de Meterología e Hidrología. En SENAMHI. 